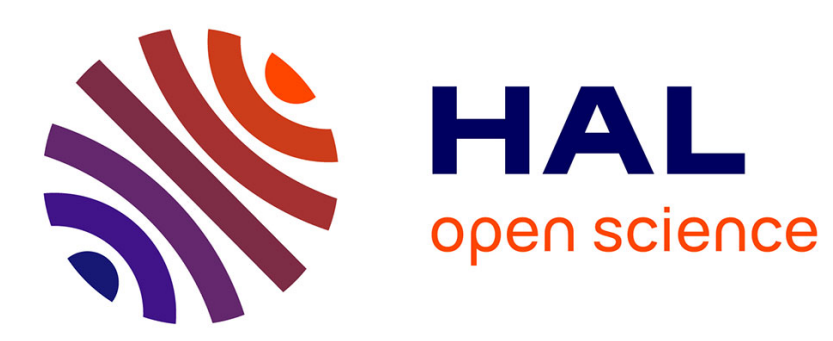

\title{
Effets de la somatotropine bovine sur les performances des vaches laitières dans les conditions françaises d'élevage
}

\author{
Y. Chilliard, R. Verite, A. Pflimlin
}

\section{> To cite this version:}

Y. Chilliard, R. Verite, A. Pflimlin. Effets de la somatotropine bovine sur les performances des vaches laitières dans les conditions françaises d'élevage. Productions Animales, 1989, 2 (5), pp.301-312. hal00895877

\section{HAL Id: hal-00895877 \\ https://hal.science/hal-00895877}

Submitted on 1 Jan 1989

HAL is a multi-disciplinary open access archive for the deposit and dissemination of scientific research documents, whether they are published or not. The documents may come from teaching and research institutions in France or abroad, or from public or private research centers.
L'archive ouverte pluridisciplinaire HAL, est destinée au dépôt et à la diffusion de documents scientifiques de niveau recherche, publiés ou non, émanant des établissements d'enseignement et de recherche français ou étrangers, des laboratoires publics ou privés. 
INRA Prod. Anim., 1989, 2 (5), 301-312
Y. CHILLIARD, R. VERITE*
A. PFLIMLIN**

INRA Laboratoire de la Lactation

Theix 63122 Saint-Genès-Champanelle

* INRA Station de Recherches

sur la Vache laitière

St-Gilles 35590 L'Hermitage

* * ITEB 149, rue de Bercy 75012 Paris

\section{Effets}

\section{de la somatotropine} bovine sur les performances des vaches laitières dans les conditions françaises d'élevage

\footnotetext{
De nombreuses expérimentations ont été réalisées pour quantifier l'effet de la somatotropine bovine sur les performances des vaches laitières. Cet article présente une étude détaillée des effets d'injections de BST sous forme retard : production et composition du lait, en relation avec la dose injectée et l'alimentation, variations de l'état corporel, reproduction et santé des animaux traités.
}

\begin{abstract}
Les connaissances sur les effets de la BST somatotropine bovine ou hormone de croissance - se sont rapidement accrues depuis 1985 en raison de sa production par les techniques de recombinaison génétique. Les études sur la BST ont en effet commencé au cours des années 30 , en utilisant la BST hypophysaire plus ou moins purifiée. La nécessité d'extraire la BST à partir d'hypophyses prélevées dans les abattoirs a toutefois fortement limité le nombre
\end{abstract}

\section{Résumé}

Vingt essais ont été réalisés en France sur 360 vaches recevant de la somatotropine (BST) retard tous les 14 ou 28 jours, à des doses correspondant à 24 ou $35 \mathrm{mg} / \mathrm{j}$. Les données obtenues suggèrent, en première approximation, que l'effet de la BST retard sur la production laitière varie assez nettement selon la dose totale injectée chaque mois, et assez faiblement selon la fréquence d'injection ( 1 ou 2 fois par mois). Selon les conditions d'utilisation (dose et fréquence), les effets moyens ont été compris entre $+2,2$ et $+3,7 \mathrm{~kg}$ de lait par jour avec des rations hivernales, et entre $+1,2$ et $+2,3 \mathrm{~kg}$ de lait par jour au pâturage. Les réponses inférieures au pâturage pourraient être dues à la qualité de l'herbe, au stade de gestation ou à la durée du traitement.

La composition du lait n'est pas modifiée au pâturage chez des vaches en milieu de lactation. Il existe une tendance à la diminution du taux protéique $(-0,5 \mathrm{~g} / \mathrm{l}$ environ) avec les rations hivernales distribuées lorsque le traitement à la BST est de 3 à 4 mois après le pic de lactation. Ces chiffres provisoires devront être réévalués sur la base d'un plus grand nombre d'essais et après dépouillement statistique approfondi. Outre ces valeurs moyennes, la production et la composition du lait fluctuent entre deux injections successives, de façon croissante lorsque s'accroît l'intervalle entre injections.

Les vaches traitées montrent aussi une diminution de leur note d'état corporel (0,3 point environ) lorsqu'elles reçoivent une ration hivernale au pic de lactation (ce qui peut entraîner un retard de fécondation non négligeable), ou lorsqu'elles sont au pâturage avec des quantités limitées de concentré. La variabilité de la réponse est très grande entre essais et entre vaches. Les données actuellement publiées ne permettent pas d'évaluer d'éventuels effets à long terme sur la santé et la carrière des animaux. et la durée des études. Depuis les années 80, la production, par les techniques de génie génétique et de fermentation bactérienne, de BST recombinée a permis des études à long terme et à assez grande échelle, en injectant chaque jour le produit à chaque vache traitée. Depuis 1985 , les progrès dans la préparation de formulations de BST à action prolongée (BST « retard ») ont permis d'expérimenter en injectant le produit tous les 14 ou tous les 28 jours. Les différentes firmes produisent des BST de structures moléculaires légèrement différentes et gardées confidentielles, et les excipients utilisés pour formuler la BST retard sont aussi du domaine privé.

Une analyse de l'ensemble des résultats disponibles en septembre 1988 a été présentée à la Commission des Communautés Européennes (Chilliard 1988b), concernant 31 essais américains et 12 essais européens, dont 7 essais français. Il est apparu que les résultats obtenus dans les conditions européennes d'élevage sont sensiblement inférieurs à ceux obtenus outreatlantique, ce qui justifie une étude plus détaillée de l'ensemble des résultats obtenus en France et disponibles au 1er janvier 1989.

\section{Les résultats obtenus à l'étranger}

\section{1 / Effets à court terme}

Les résultats de 18 essais d'une durée inférieure ou égale à 3 semaines (voir Chilliard 1988a), effectués après le pic de lactation avec des injections journalières, montrent un effet moyen de $+4,0( \pm 1,3) \mathrm{kg}$ de lait/j, avec des diminutions de l'ingestion de matière sèche $(-0,5 \pm 1,1 \mathrm{~kg} / \mathrm{j})$ et du bilan énergétique cal- 
Tableau 1. Effets à court terme (5 à 21 j) de la BST sur les performances des vaches laitières en fonction de leur bilan énergétique calculé (BEC) (d'après Chilliard 1988 a).

* Traité-témoin : moyenne ( \pm écart-type).

\begin{tabular}{|c|c|c|c|c|c|c|}
\hline $\begin{array}{l}\text { BEC des vaches traitées } \\
\text { (UFL/j) }\end{array}$ & $(\mathrm{kg} / \mathrm{j})$ & $(\%)$ & $\underset{\substack{\text { Taux } \\
\text { butyreux } \\
(\mathrm{g} / \mathrm{l})}}{*}$ & $\underset{\text { protéique }^{\text {Taux }}}{(g / l)}$ & $\begin{array}{c}\text { Matière sèche } \\
\text { ingérée } \\
\text { (kg/i) }\end{array}$ & $\begin{array}{c}\text { BEC }^{\star} \\
(\mathrm{UFL} / \mathrm{j})\end{array}$ \\
\hline $\begin{array}{c}1.9 \\
( \pm 0,8)\end{array}$ & $\begin{array}{l}+4,5 \\
( \pm 1,6)\end{array}$ & $\begin{array}{r}+19,6 \\
( \pm 7,9)\end{array}$ & $\begin{array}{l}+0,8 \\
( \pm 2,3)\end{array}$ & $\begin{array}{c}-0,8 \\
{[ \pm 0,8)}\end{array}$ & $\begin{array}{c}-0,2 \\
( \pm 1,1)\end{array}$ & $\begin{array}{c}-2,2 \\
( \pm 1,4)\end{array}$ \\
\hline $\begin{array}{l}-2,1 \\
( \pm 2,3)\end{array}$ & $\begin{array}{l}+3,5 \\
( \pm 0,7)\end{array}$ & $\begin{array}{r}+17,3 \\
( \pm 8,6)\end{array}$ & $\begin{array}{c}+3,1 \\
( \pm 2,5)\end{array}$ & $\begin{array}{l}-2,1 \\
( \pm 1,7)\end{array}$ & $\begin{array}{c}-0,8 \\
( \pm 1,0)\end{array}$ & $\begin{array}{l}-2,8 \\
( \pm 1,1)\end{array}$ \\
\hline
\end{tabular}

(1) 9 essais, 47 vaches, BEC des vaches traitées $>0,6 \mathrm{UFL} / \mathrm{j}$.

(2) 9 essais, 46 vaches, BEC des vaches traitées $<0,2$ UFL/j.

Tableau 2. Effet de la dose et de la fréquence des injections de BST sur la production laitière. Daprès la revue de Chilliard (1988b). sans tenir compte des données obtenues en France. Les données disponibles étaient exprimées soit en lait brut, soit en lait corrigé à 35 ou $40 \mathrm{~g} / \mathrm{l}$ de taux butyreux.

\begin{tabular}{|l|c|c|c|c|c|c|c|}
\hline Dose (mg/j) & $\mathbf{1 0 - 1 5}$ & $\mathbf{2 0 - 2 7}$ & $\mathbf{3 1 - 5 0}$ & $\mathbf{1 1}$ & $\mathbf{2 3}$ & $\mathbf{3 4}$ & $\mathbf{3 6}$ \\
\hline $\begin{array}{l}\text { Fréquence des injections } \\
\text { (jours) }\end{array}$ & 1 & 1 & 1 & 28 & 28 & 28 & 14 \\
\hline Nombre d'essais & 16 & 20 & 13 & 7 & 8 & 8 & 11 \\
\hline Nombre de vaches traitées & 190 & 300 & 158 & 80 & 88 & 87 & 379 \\
\hline Moyenne $^{\star}(\mathrm{kg} / \mathrm{j})$ & 3,9 & 5,2 & 5,6 & 2,7 & 4,1 & 4,8 & 4,6 \\
\hline Ecart-type & 2,1 & 2,1 & 2,4 & 1,5 & 1,1 & 1,4 & 2,0 \\
\hline
\end{tabular}

* (traité-témoin), moyenne pondérée par le nombre de vaches traitées.

culé $(-2,5 \pm 1,2 \mathrm{UFL} / \mathrm{j})$. La réponse en lait était plus faible pendant les 2 premiers mois de lactation qu'après le pic de lactation.

Les effets sur la composition du lail dépendent du bilan énergétique des vaches traitées (tableau 1): peu de variation avec la BST si le bilan reste positif, mais augmentation du taux butyreux $(+3 \mathrm{~g} / \mathrm{l})$ et diminution du taux protéique $(-2 \mathrm{~g} / 1)$ si le bilan devient négatif. Ce phénomène est analogue à ce qui est observé chez des vaches non traitées en bilan négatif (cf Journet et Chilliard 1985, Rémond 1985).

\section{2 / Effets à long terme (voir Chilliard 1988b)}

\section{a / Production et composition du lait}

Avec des injections journalières les effets obtenus dans 13 à 20 essais avec des doses croissantes de BST varient de $+3,9$ à $+5,6 \mathrm{~kg}$ de lait/j (tableau 2), mais avec des réponses très variables entre essais (de 0 à $+11 \mathrm{~kg} / \mathrm{j}$ ). L'injection de BST retard lous les 14 ou 28 jours donne des résultats inférieurs (d'environ 15-30\%, à dose totale comparable). La fréquence d'injection modifie peu le résultat si la dose journalière moyenne est la même (tableau 2). La variabilité des réponses entre troupeaux ou essais (comprises entre +1 et $+8 \mathrm{~kg} / \mathrm{i}$ ) est, là encore, très élevée.

La composition du lait n'a pas varié en moyenne sur les périodes d'études, mais a montré des fluctuations plus ou moins impor- tantes durant l'intervalle de temps séparant deux injections de BST retard (ce point est développé plus loin).

L'effet de la BST retard sur la courbe de lactation est illustré par la figure 1. Dans cet essai, représentatif de la tendance générale, les vaches ont reçu la même ration, à base de fourrages conservés, pendant toute la lactation. On observe une réponse immédiate de la production laitière $(+4,5 \mathrm{~kg} / \mathrm{j})$, qui diminue légèrement au cours de la période de traitement pour atteindre $+2,5 \mathrm{~kg} / \mathrm{j}$ pendant les dernières semaines.

\section{$b$ / Ingestion de la ration et bilan énergétique des animaux}

L'ingestion n'augmente significativement qu'après un délai de 6 à 8 semaines (figure 1). Les augmentations sont d'environ $+1,5 \mathrm{~kg}$ MS/j dans les essais qui durent 32 semaines.

Elles sont seulement d'environ $+0,8 \mathrm{~kg} \mathrm{MS} / \mathrm{j}$ dans les essais qui ne durent que 18 semaines, en raison du poids plus important des 6-8 premières semaines dans la réponse moyenne, et éventuellement d'autres facteurs (fréquence des injections, régimes alimentaires, etc).

Il résulte de ces évolutions respectives de la production laitière et de l'ingestion de matière sèche, que les vaches traitées sont tout d'abord en bilan énergétique plus faible que les vaches témoins (figure 1). Dans la deuxième phase du traitement elles sont toutefois en mesure d'inverser la tendance, tout au moins lorsqu'elles 
Figure 1. Effets de la BST retard (500 $\mathrm{mg}$ tous les 14 jours) sur la production, la consommation et le bilan énergétique de vaches recevant une ration complète à volonté (Phipps 1987).
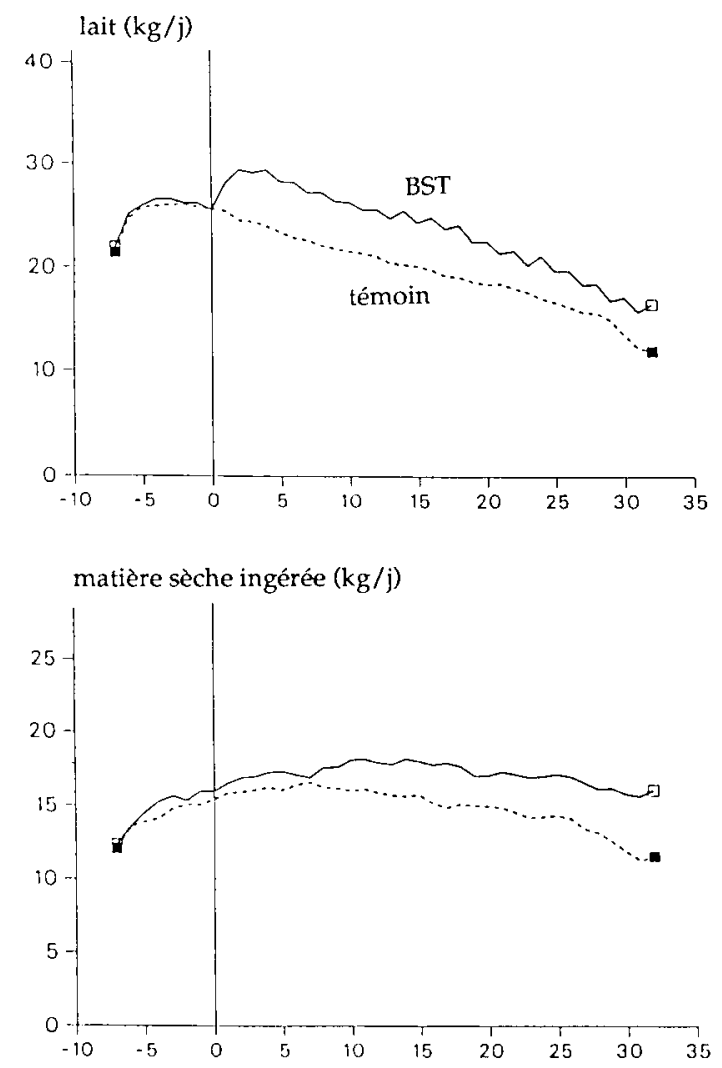

bilan énergétique calculé (MJ/j)

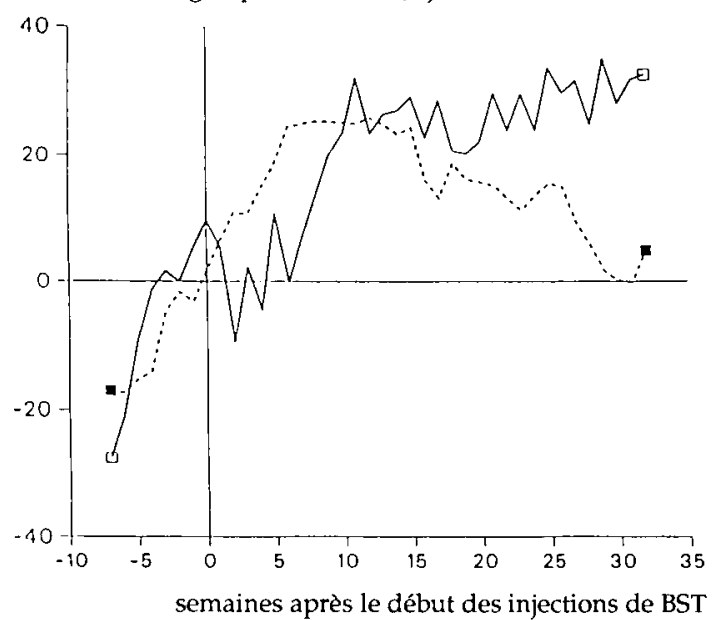

disposent d'une ration très ingestible à haute valeur nutritionnelle (bons fourrages et proportion élevée de concentré). Dans 8 essais portant sur 315 vaches traitées, et recevant ce type de régime, le bilan énergétique par rapport au lot témoin a été inférieur de 1,2 UFL/j pendant les 12 premières semaines de traitement, et a été supérieur de $0,7 \mathrm{UFL} / \mathrm{j}$ pendant les 22 dernières semaines. Ceci correspond théoriquement à un dépôt de lipides corporels diminué de $19 \mathrm{~kg}$ en 12 semaines, avec rattrapage pendant les 22 semaines suivantes.

\section{Les mécanismes d'action de la BST}

La somatotropine bovine (= BST), ou hormone de croissance $(=\mathrm{GH}=$ growth hormone), est un polypeptide sécrété par l'hypophyse. Oulre ses effets stimulants sur la croissance osseuse et l'anabolisme protéique, on connaît depuis plusieur's décennies ses effels galactopoiéliques, c'est-à-dire l'augmentation de la production lailière au cours d'une lactation déjà établie.

L'effet déterminant de la BST s'exerce sans doute au niveau du tissu mammaire. Cet effet est toutefois indirect, car la BS'T n'agit pas sur le lissu mammaire isolé in vitro, ni in vivo lorsqu'elle est infusée dans la mamelle. La BST stimule en réalité la production de messagers, tels que les somatomédines ( = IGFs = Insulin like Growth Factors) produiles par le foie el différents tissus qui sont probablement à l'origine de la plupart des effets anaboliques de la BST: croissance osseuse el musculaire, svnthèse des constituants du lait. réduction du catabolisme azoté.

On sait que la sous-alimentalion réduil la synthèse des somatomédines, ce qui explique peutêtre que les réponses à la BST soient plus fábles chez un animal sous-alimenté.

Par ailleurs, la BST accroît l'effet des hormones lipolytiques sur les tissus adipeux, et froine l'effet lipogénique de linsuline, ce qui permet d'augmenter lapport de substrats énergétiques nécessaires à la mamelle, et la participation des lipides corporels dans le métabolisme intermédiaire. Il en résulte une substantielle épargne de glucose (plus de la moilié du besoin supplémenlaire de la mamelle)

La réponse du niveau d'ingestion aux injections de BST est limitée par la lenteur des adaptations digestives propres aux ruminants, et n'intervient que plus tardivement. Flle est stimulée par le drainage des nutriments par la mamelle et par d'autres modificalions métaboliques.

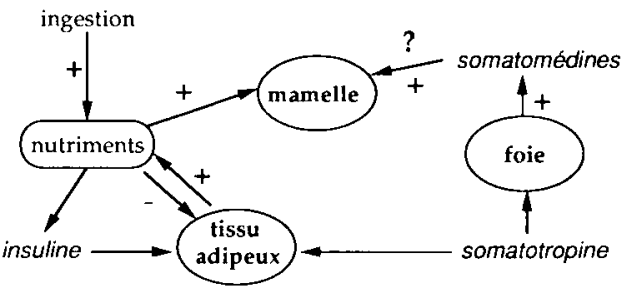

La somatotropine exogène permet un accroissement imporlant du niveau de production laitière parle biais de modifications coordonnées du métabolisme dans l'ensemble des tissus et organes de la vache (d'après Peel et Bauman 1987, Chilliard 1988d, Mc: Bride? el al 1988).

Ceci est confirmé, en moyenne sur l'ensemble des résultats disponibles, par les évolutions de composition corporelle (estimée à l'aide de l'eau lourde), de poids vif, de notes d'état corporel el de métabolites sanguins. Il faut toutefois souligner que lorsque la ration a une teneur plus faible en énergie, ou lorsque les traitements durent moins de 32 semaines, les vaches traitées sont en moins bon état corporel à la fin de l'expérience, et devront recevoir ulté- 
Tableau 3. Récapitulatif (au 01.01.1989) des essais BST retard en France.

$P R=$ Pie Rouges $; N O=$ Normandes $;{ }^{*}=$ Résultats provisoires $;{ }^{\star \star}=$ Période hivernale seulement $; N C=$ Non communiqué

\begin{tabular}{|c|c|c|c|c|c|c|c|c|c|c|c|}
\hline \multirow{2}{*}{$\frac{\begin{array}{c}\text { Dose } \\
(\mathrm{mg} / \mathrm{j})\end{array}}{23 \text { ou } 25}$} & \multirow[b]{2}{*}{$\begin{array}{c}\text { Essais } \\
1 \\
2 \\
3 \\
4 \\
5 \mathrm{PR} \\
6^{*} \\
7 \mathrm{NO} \\
8^{\star} \\
9^{*} \\
10 \mathrm{PR}^{*} \\
11^{*}\end{array}$} & \multirow{2}{*}{$\begin{array}{c}\begin{array}{c}\text { N. vaches } \\
\text { traitées }\end{array} \\
10 \\
12 \\
32 \\
10 \\
16 \\
12 \\
20 \\
23 \\
14 \\
10 \\
14\end{array}$} & \multirow{2}{*}{$\begin{array}{c}\begin{array}{c}\text { Semaines } \\
\text { BST }\end{array} \\
24 \\
20 \\
16 \\
12 \\
20 \\
28 \\
24 \\
32 \\
32 \\
32 \\
32\end{array}$} & \multirow{2}{*}{$\begin{array}{c}\text { Semaines } \\
\text { R. Hiv. (a) } \\
24 \\
8 \\
16 \\
0 \\
8 \\
12 \\
8 \\
12 \\
10 \\
12 \\
18\end{array}$} & \multicolumn{2}{|c|}{$\begin{array}{c}\text { Lait }(\mathrm{kg} / \mathrm{j}) \\
\mathrm{B}-\mathrm{T}(\mathrm{b})\end{array}$} & \multirow{2}{*}{\begin{tabular}{|c|} 
T. BUT. $(\mathbf{g} / \mathbf{l})$ \\
B-T \\
0,1 \\
$-0,1$ \\
1,2 \\
$-0,4$ \\
$-1,2$ \\
0,3 \\
$-1,8$ \\
0,0 \\
$-0,2$ \\
0,7 \\
$-0,6$
\end{tabular}} & \multirow{2}{*}{$\begin{array}{c}\text { T. PROT. } \\
\text { B-T } \\
0,5 \\
-0,2 \\
-0,8 \\
0,2 \\
-0,3 \\
-0,4 \\
-1,0 \\
0,0 \\
-0,5 \\
-0,5 \\
-0,2\end{array}$} & \multirow{2}{*}{$\begin{array}{c}\text { Etat CORP } \\
\text { B-T (c) } \\
-0,3 \\
0,0 \\
-0,1\end{array}$} & \multicolumn{2}{|c|}{$\begin{array}{c}\begin{array}{c}\text { Concentré } \\
(\mathrm{kg} / \mathrm{j})\end{array} \\
\mathrm{T}^{\mathrm{B}-\mathrm{T}}\end{array}$} \\
\hline & & & & & $\begin{array}{l}24,8 \\
21,0 \\
27,1 \\
16,3 \\
17,7 \\
24,6 \\
17,0 \\
18,7 \\
23,3 \\
18,9 \\
23,9\end{array}$ & $\begin{array}{l}2,7 \\
1,2 \\
2,7 \\
0,1 \\
1,3 \\
2,8 \\
1,6 \\
2,0 \\
0,9 \\
1,9 \\
1,7\end{array}$ & & & & $\begin{array}{l}4,9 \\
3,3 \\
6,2 \\
1,2 \\
2,2 \\
\\
1,9 \\
2,6\end{array}$ & $\begin{array}{l}0,4 \\
0,0 \\
0,0 \\
0,1 \\
0,2 \\
\\
0,3 \\
0,4\end{array}$ \\
\hline \multicolumn{2}{|c|}{$\begin{array}{l}\text { Moyenne (d) } \\
\text { Ecart-type }\end{array}$} & $\begin{array}{r}16 \\
7\end{array}$ & $\begin{array}{r}24 \\
7\end{array}$ & & $\begin{array}{r}21,2 \\
3,7\end{array}$ & $\begin{array}{l}1,8 \\
0,9\end{array}$ & $\begin{array}{r}-0,1 \\
0,9\end{array}$ & $\begin{array}{r}-0,4 \\
0,4\end{array}$ & $\begin{array}{r}-0,3 \\
0,2\end{array}$ & & \\
\hline \multicolumn{2}{|c|}{$\begin{array}{l}\text { Moyenne (d) } \\
\text { Ecart-type }\end{array}$} & $\begin{array}{r}17 \\
6\end{array}$ & $\begin{array}{l}22 \\
10\end{array}$ & & $\begin{array}{r}21,0 \\
3,0\end{array}$ & $\begin{array}{l}2,7 \\
1,2\end{array}$ & $\begin{array}{l}0,7 \\
1,0\end{array}$ & $\begin{array}{l}0,2 \\
0,5\end{array}$ & $\begin{array}{r}-0,3 \\
0,4\end{array}$ & & \\
\hline
\end{tabular}

(a) Période d'alimentation à l'étable avec une ration à base de fourrages conservés.

(b) Lot BS'T - Lot témoin.

(c) Note d'état corporel (échelle de 0 à 5 ).

(d) Moyenne pondérée par le nombre de vaches traitées dans chaque essai, et écart-type inter-essais.

rieurement un supplément d'énergie pour arriver au vêlage suivant dans un état comparable.

\section{c / Efficacité d'utilisation de la ration}

Les études effectuées en chambres respiratoires et les essais zootechniques montrent que la BST ne modifie ni le besoin d'entretien ni l'efficacité d'utilisation digestive et métabolique de l'énergie ingérée lorsque les vaches sont maintenues à poids vif et état corporel constants (voir aussi Kirchgessner et al 1989). Le besoin énergétique total augmente donc normalement avec le supplément de lait produit et correspond aux recommandations habituelles de l'INRA.

Le rapport «lait produit/énergie nette ingérée (UFL) » augmente en fonction de la diminution de la part du besoin d'entretien (fixe) dans le besoin total (qui augmente), soit $+2,6 \%$ ou $+4,4 \%$ pour des réponses en lait de +2 ou $+4 \mathrm{~kg} / \mathrm{j}$, respectivement. Lorsque l'efficacité apparente augmente plus fortement, c'est en raison d'une diminution de l'état corporel des vaches traitées par rapport aux témoins, qui devra être récupéré après le traitement.

\section{d / Réponses en fonction du numéro de lactation et du potentiel de production}

La réponse des primipares (en $\mathrm{kg}$ de lait par jour) a été comparable à celle des multipares dans 5 essais, et inférieure dans 5 autres essais. Lorsque les chercheurs ont voulu analyser la réponse en fonction du potentiel du troupeau ou des vaches individuelles, ils ont observé soit une absence de relation ( 3 études), soit une réponse inférieure chez les plus fortes productrices (2 études).

\section{Les résultats obtenus en France}

\section{1 / Les essais}

Le dossier qui nous a été confié comportait les résultats de 20 essais effectués en France sur 360 vaches traitées avec la BST retard. Un autre essai a été effectué avec la BST en injections journalières - il ne sera pas utilisé dans ce qui suit - il confirme, sur 14 vaches traitées recevant une ration hivernale pendant 30 semaines, les tendances observées à l'étranger 
(réponse égale à $69 \%$ de la réponse moyenne obtenue avec le même traitement à l'étranger, cf tableau 2).

Les résultats proviennent d'essais réalisés avec 3 sortes de BST retard, et les fréquences d'injection ont été soit de 14 , soit de 28 jours. Pour une même dose totale, les résultats sont voisins avec ces 2 fréquences d'injection si l'on compare d'un côté 5 essais sur 77 vaches, et de l'autre 6 essais sur 96 vaches. Ceci a aussi été confirmé dans le même essai (mêmes conditions expérimentales) par Vérité et al (1989).

Pour cette raison, les résultats seront présentés en fonction de la dose moyenne injectée quotidiennement, sans tenir compte de la fréquence d'injection (sauf pour les fluctuations de composition du lait, $\mathrm{cf} 4$ ). Il faut toutefois souligner la nécessité de disposer d'un plus grand nombre de comparaisons directes des 2 fréquences d'injections pour conclure avec certitude sur leurs effets respectifs.

Cinq essais ont été réalisés sur des troupeaux INRA, 3 essais ont été réalisés par l'Enseignement Supérieur Agronomique, 8 essais par l'ITEB (dont 5 essais conduits au pâturage, et dépouillés par Bertrand et al 1989), et 4 essais proviennent de troupeaux privés. Onze essais ont été réalisés dans des troupeaux expérimentaux, et $\mathbf{9}$ en fermes commerciales (la plupart ont été choisies parmi les troupeaux adhérents au contrôle laitier, ayant un bon niveau de production et un bon état sanitaire, une taille supérieure à 50 vaches et une bonne maîtrise de la production fourragère). Les consommations individuelles des vaches en période hivernale ne sont connues que dans 4 essais réalisés à l'INRA. L'Ouest, l'Est, le Sud-Ouest, le SudEst et le Centre de la France sont représentés. Quinze essais ont été réalisés sur vaches de race Pie-noire, 4 sur Pie-rouge (dont 2 sur Montbéliarde), et 2 sur Normande.

Treize essais ont duré au moins 20 semaines, et 6 essais de courte durée (12 semaines) n'ont concerné que la période de pâturage (tableau 3).

La plupart des essais comparent 2 lots d'animaux : lot BST et lot témoin (recevant éventuellement un placebo). Quelques essais comparent plusieurs doses de BST ou fréquences d'injections $\left(\mathrm{n}^{\circ} 1,3,4\right)$, ou étudient les interactions entre la BST et la nature de la ration $\left(n^{\circ} 3,20\right)$.
Dans la plupart des essais de longue durée (plus de 20 semaines), on a étudié successivement une alimentation hivernale puis une période de pâturage. En période hivernale, toutes les rations étaient à base d'ensilage de maïs distribué à volonté (avec d'autres fourrages en quantités limitées dans les essais 5,7 , $9,10,11,12,13$ et 19$)$. Le concentré était distribué en fonction du niveau de production laitière, avec des rythmes d'apport variables selon les essais (voir plus loin), mais représentatifs des pratiques habituelles en France.

Les mesures et contrôles effectués et communiqués avec le dossier sont très hétérogènes entre essais, ce qui ne permet pas un dépouillement de tous les paramètres sur un nombre important d'essais et de vaches. Par ailleurs, certains résultats (5 essais) sont provisoires. Il en découle que les tendances rapportées doivent être considérées avec la plus grande prudence, et sont susceptibles d'être revues lorsque tous les résultats seront publiés et analysés statistiquement.

\section{2 / Résultats globaux}

Les conditions expérimentales et résultats principaux des 20 essais sont décrits dans le tableau 3, en séparant les 2 doses utilisées. Soulignons en premier lieu la grande variabilité entre troupeaux des réponses moyennes: de $0 \grave{a}+3 \mathrm{~kg} / \mathrm{j}$ pour $24 \mathrm{mg} / \mathrm{j}$ de BST retard, et de 0 à $+4 \mathrm{~kg} / \mathrm{j}$ pour $35 \mathrm{mg} / \mathrm{j}$. Les 2 doses ont été étudiées sur des effectifs et pendant des durées comparables.

Les résultats moyens sont de $+1,8 \mathrm{~kg} / \mathrm{j}$ de lait avec $24 \mathrm{mg}$ de BST $/ \mathrm{j}$ et $+2,7 \mathrm{~kg} / \mathrm{j}$ avec $35 \mathrm{mg}$, sans modifications importantes de la composition du lait, mais avec une tendance fréquente à la diminution de la note d'état corporel. Le rapport des réponses obtenues avec les deux doses est de $\mathbf{0 , 6 7}$ dans les essais français, alors qu'il était de 0,85 à 0,93 dans les essais réalisés à l'étranger (cf tableau 2).

\section{a / en période d'alimentation hivernale}

Avec la dose de $24 \mathrm{mg} / \mathrm{j}$ (tableau 4), la réponse en lait est de $2,2( \pm 0,7) \mathrm{kg} / \mathrm{j}$ dans 10 essais pendant 13 semaines en moyenne, avec une diminution de $0,6 \mathrm{~g} / \mathrm{l}$ du taux protéique.

Tableau 4. Résultats obtenus en alimentant les vaches à l'étable, avec une ration à base de fourrages conservés

\begin{tabular}{|c|c|c|c|c|c|c|c|}
\hline $\begin{array}{c}\text { Dose BST } \\
(\mathrm{mg} / \mathrm{j})\end{array}$ & & $\begin{array}{c}\text { Lait } \\
\mathrm{kg} / \mathrm{j}(\%)\end{array}$ & $\begin{array}{c}\text { TB } \\
(g / 1)\end{array}$ & $\begin{array}{c}\text { TP } \\
(\mathrm{g} / \mathrm{l})\end{array}$ & $\begin{array}{c}\text { MSI } \\
(\mathrm{kg} / \mathrm{j})\end{array}$ & $\begin{array}{c}\text { BEC } \\
\mathrm{UFL} / \mathrm{j}\end{array}$ & $\begin{array}{c}\text { Variation } \\
\text { de note } \\
\text { d'état }\end{array}$ \\
\hline 24 & $\begin{array}{l}\text { Nombre d'essais } \\
\text { Nombre de vaches traitées } \\
\text { Réponse * : } \\
\text { Moyenne } \\
\text { Ecart-type }\end{array}$ & $\begin{array}{c}10 \\
159 \\
+2,2(+9 \%) \\
\pm 0,7( \pm 3 \%)\end{array}$ & $\begin{array}{r}9 \\
134 \\
+\quad 0,1 \\
\pm 1,0\end{array}$ & $\begin{array}{r}9 \\
134 \\
-0,6 \\
\pm 0,5\end{array}$ & $\begin{array}{r} \\
\quad 79 \\
+\quad 0,2 \\
\pm 0,5\end{array}$ & $\begin{array}{r}3 \\
54 \\
-0,7 \\
\pm 0,5\end{array}$ & $\begin{aligned} & 3 \\
& 54 \\
& \\
- & 0,2 \\
\pm & 0,1\end{aligned}$ \\
\hline 35 & $\begin{array}{l}\text { Nombre d'essais } \\
\text { Nombre de vaches traitées } \\
\text { Réponse* }^{\text {Moyenne }} \\
\text { Ecart-type }\end{array}$ & $\begin{array}{c}5 \\
91 \\
+3,7(+17 \%) \\
\pm 1,4( \pm 9 \%)\end{array}$ & $\begin{array}{r}5 \\
91 \\
-0,2 \\
\pm 1,2\end{array}$ & $\begin{array}{r}5 \\
91 \\
-0,4 \\
\pm 0,4\end{array}$ & $\begin{array}{r}5 \\
91 \\
+\quad 0,6 \\
\pm 0,6\end{array}$ & $\begin{array}{r}4 \\
73 \\
-0,9 \\
\pm 0,8\end{array}$ & $\begin{array}{r}4 \\
70 \\
-0,2 \\
\pm 0,2\end{array}$ \\
\hline
\end{tabular}

* Lot BST - lot témoin, moyenne pondérée par le nombre de vaches, \pm écart-type inter-essais (les chiffres entre parenthèses sont les réponses en \% du lot témoin) - TB, TP, MSI, BEC = taux butyreux et protéique, matière sèche ingérée, bilan énergétique calculé.

\section{L'effet de la BST varie avec la dose injectée : en moyenne $+1,8 \mathrm{~kg}$ lait $/ \mathrm{j}$ avec l'équivalent de $24 \mathrm{mg} \mathrm{BST/j}$ et $+2,7 \mathrm{~kg} \mathrm{lait} / \mathrm{j}$ avec l'équivalent de $35 \mathrm{mg} \mathrm{BST} / \mathrm{j}$.}


Dans 4 essais où l'ingestion a été contrôlée, l'ingestion de la ration totale a augmenté de 0,2 $\mathrm{kg}$ de MS/j par rapport au témoin, et le bilan énergétique a diminué $(-0,7 \mathrm{UFL} / \mathrm{i})$, ainsi que le poids vif $(-10 \mathrm{~kg}$ dans 6 essais sur 102 vaches).

Avec la dose de $35 \mathrm{mg} / \mathrm{j}$ (tableau 4), la réponse moyenne dans 5 essais est de $3,7 \mathrm{~kg} / \mathrm{j}$ ( $\pm 1,4 \mathrm{~kg}$ ) pendant 23 semaines en moyenne. On observe simultanément une baisse du taux protéique $(-0,4 \mathrm{~g} / \mathrm{l})$ et du bilan énergétique (- 0,9 UFL/i), l'ingestion ayant augmenté de 0,6 $\mathrm{kg} \mathrm{MS} / \mathrm{j}$. Il n’a malheureusement pas été possible de connaître les quantités de concentré distribuées dans 2 des essais ayant conduit aux réponses les plus élevées ( $\mathrm{n}^{0} 12$ et 13).

Dans 6 troupeaux d'Europe du Nord (2 aux Pays Bas, 2 en Angleterre, 2 en Allemagne) recevant des rations riches en concentrés et 35 $\mathrm{mg} / \mathrm{j}$ de BST retard, la réponse moyenne est de $+3,8( \pm 0,8) \mathrm{kg} / \mathrm{j}$ (cf Chilliard 1988b, Lebzien et al 1989).

La réponse à l'injection de BST évolue rapidement : la quantité de lait est augmentée pendant les 10 premiers jours qui suivent chaque injection, puis elle diminue. Les taux butyreux et protéique varient aussi pendant la même période, avec des amplitudes qui peuvent être importantes.

\section{b / au pâturage}

Avec la dose de $24 \mathrm{mg} / \mathrm{j}$, la réponse moyenne dans 9 essais est de $1,2 \mathrm{~kg}$ de lait $/ \mathrm{j}$ ( $\pm 0,7 \mathrm{~kg}$ ) (tableau 5) pendant 13 semaines en moyenne. Le taux butyreux diminue de $0,6 \mathrm{~g} / \mathrm{l}$ mais le taux protéique ne varie pas.

Avec la dose de $35 \mathrm{mg} / \mathrm{j}$, la réponse moyenne dans 8 essais est de $2,3 \mathrm{~kg}$ de lait $/ \mathrm{j}( \pm 1,1 \mathrm{~kg}$ ) (tableau 5) pendant 14 semaines en moyenne. Le taux butyreux augmente de $1,0 \mathrm{~g} / \mathrm{l}$ et le taux protéique de $0,3 \mathrm{~g} / \mathrm{l}$, alors que la note d'état corporel tend à diminuer (- 0,4 point)

L'augmentation du taux butyreux est plus nette au début des périodes d'injection (6

Figure 2. Evolution journalière de la production et de la composition du lait après des injections mensuelles de BST retard (Vérité et al 1989, essai 2). La valeur 100 représente la moyenne des valeurs observées durant les jours - 7 à - 1 et $+22 \dot{a}+28$ au cours de 5 périodes d'injection.

\section{Réponse relative (\%)}

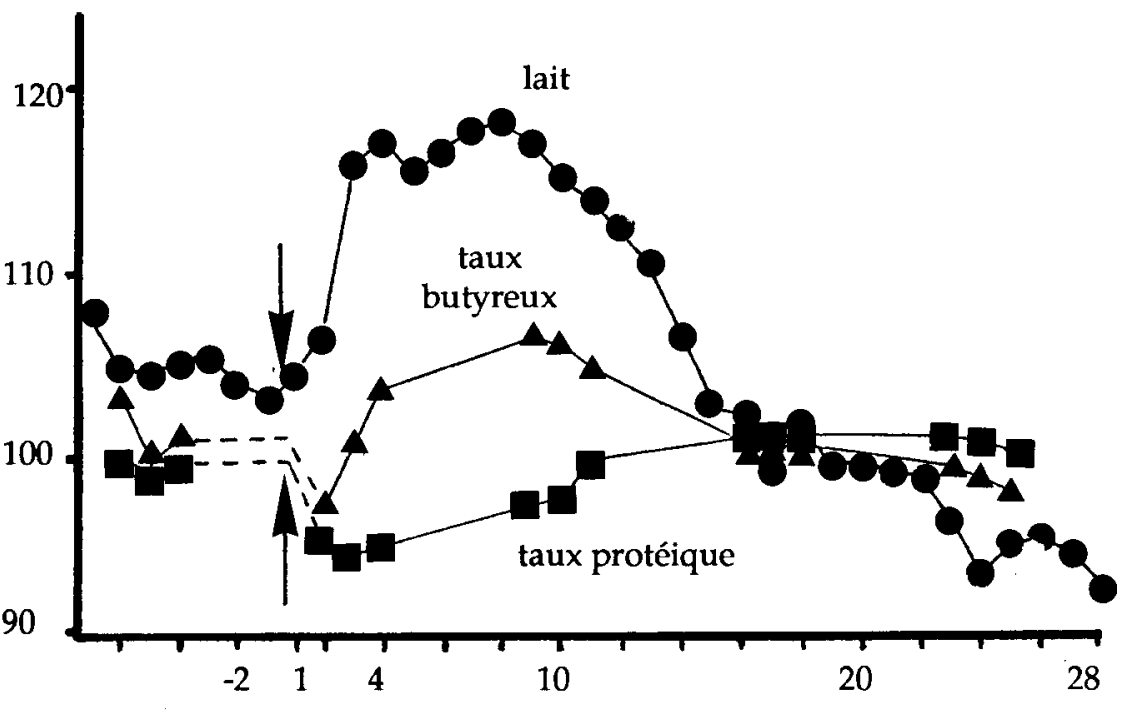

jours après injection semaines environ), dans les essais qui débutent au pâturage. Ceci correspond probablement à une sous-alimentation énergétique, avant que l'ingestion d'herbe n'augmente.

Dans la plupart des essais conduits au pâturage les animaux ont reçu un complément de fourrages conservés (généralement de l'ensilage de maïs). Malgré cela, on constate des diminutions de note d'état corporel, y compris dans les essais de longue durée $\left(\mathrm{n}^{\circ} \mathbf{8}, 9,10,20\right.$, tableau 3 ). La reconstitution des réserves est donc probablement plus difficile au pâturage qu'avec un régime ensilage de maïs distribué toute l'année. Dans les conditions françaises où le pâturage est le système d'affouragement estival le plus fréquent; un traitement à la BST pourrait se traduire par un amaigrissement des vaches vêlant à l'automne, à moins qu'elles ne reçoivent des suppléments d'ensilage de maïs ou d'aliments concentrés en fin de lactation, voire après le tarissement.

\section{3 / Variations de composition corporelle}

Des estimations de la composition corporelle ont été effectuées en utilisant l'eau lourde dans trois essais INRA ( $\mathbf{n}^{\circ} 1,2$ et 20 ). Les résultats de deux essais sont actuellement disponibles (tableau 6). Ils montrent que les vaches traitées mobilisent plus, ou déposent moins, de lipides corporels que les vaches témoins, avec un régime hivernal ou au pâturage. Ces résultats sont pour l'essentiel en accord avec les évolutions des notes d'état corporel, des bilans énergétiques calculés, et des teneurs en acides gras non estérifiés plasmatiques.

\section{4 / Fluctuation de la réponse entre 2 injections}

A la suite de chaque injection mensuelle, on assiste à des réponses cycliques de la production et de la composition du lait, avec généralement un maximum de production laitière et de taux butyreux entre 5 et 10 jours après chaque injection, alors que le taux protéique est minimum dès le lendemain et pendant une semaine environ, puis augmente au cours de la semaine suivante (figure 2). L'accroissement du taux butyreux est parallèle à une élévation du taux plasmatique d'acides gras non estérifiés, et suit celui de la production laitière. Il s'accompagne d'un enrichissement de la matière grasse du lait en acides gras à longue chaîne (stéarique et surtout oléique) provenant des lipides corporels (cf Vérité et al 1989, Chilliard 1988b). La diminution du taux protéique (et de l'urémie sanguine), qui précède l'augmentation de production laitière est difficile à expliquer par un effet de dilution (lait) ou de sous-alimentation (animal). Elle pourrait être liée à l'effet d'épargne azotée de la BST sur les protéines corporelles et l'oxydation des acides aminés (cf Chilliard 1988a), ce qui serait compatible avec l'augmentation du taux protéique en fin de cycle, lorsque la production laitière diminue rapidement.

L'amplitude des variations de composition du lait peut être importante dans le cas d'injections tous les 28 jours : de 2 à $5 \mathrm{~g} / \mathrm{l}$ pour le taux butyreux et de 1 à $2 \mathrm{~g} / \mathrm{l}$ pour le taux protéique (cf figure 2 et autres essais). La réponse en lait 
Tableau 5. Résultats obtenus au pâturage.

\begin{tabular}{|c|c|c|c|c|c|}
\hline $\begin{array}{c}\text { Dose BST } \\
(\mathrm{mg} / \mathrm{j})\end{array}$ & & $\underset{\mathrm{kg} / \mathrm{j}(\%)}{\text { Lait }}$ & $\begin{array}{c}\text { TB } \\
(\mathrm{g} / \mathrm{l})\end{array}$ & $\underset{(\mathrm{g} / \mathrm{l})}{\mathrm{TP}}$ & $\begin{array}{c}\text { Variation } \\
\text { de note } \\
\text { d'état }\end{array}$ \\
\hline 24 & $\begin{array}{l}\text { Nombre d'essais } \\
\text { Nombre de vaches traitées } \\
\text { Réponse * : }^{\text {Moyenne }} \\
\text { Ecart-type }\end{array}$ & $\begin{array}{c}9 \\
143 \\
+1,2(+6 \%) \\
\pm 0,7( \pm 4 \%)\end{array}$ & $\begin{array}{r}8 \\
105 \\
-\quad 0,6 \\
\pm 0,9\end{array}$ & $\begin{array}{r}8 \\
105 \\
-0,1 \\
\pm 0,2\end{array}$ & $\begin{array}{r}1 \\
12 \\
+\quad 0,2\end{array}$ \\
\hline 35 & $\begin{array}{l}\text { Nombre d'essais } \\
\text { Nombre de vaches traitées } \\
\text { Réponse }^{*} \text { : } \\
\\
\text { Moyenne } \\
\text { Ecart-type }\end{array}$ & $\begin{array}{c}8 \\
138 \\
+2,3(+11 \%) \\
\pm 1,1( \pm 5 \%)\end{array}$ & $\begin{array}{r}8 \\
138 \\
+\quad 1,0 \\
\pm 0,8\end{array}$ & $\begin{array}{r}8 \\
138 \\
+\quad 0,3 \\
\pm 0,5\end{array}$ & $\begin{array}{r}5 \\
88 \\
-0,4 \\
\pm 0,2\end{array}$ \\
\hline
\end{tabular}

*Voir tableau 4.

Tableau 6. Variations de composition corporelle des vaches traitées par la BST (35 mg/j) (Vérité et Chilliard non publié, Chilliard et al 1989 et non publié).

Les résultats sont exprimés par différence avec le lot témoin ( 7 et 12 vaches par lot, dans les essais 1 et 20 , respectivement). La variation de lipides corporels est estimée en utilisant la méthode de l'eau lourde.

\begin{tabular}{|c|c|c|c|}
\hline $\begin{array}{c}\text { Essai } \\
\text { Régime } \\
\text { Durée (semaines) }\end{array}$ & $\begin{array}{l}\mathrm{N}^{\mathrm{N}} 1 \\
\text { Hivernal } \\
24\end{array}$ & $\begin{array}{c}\text { Hivernal } \\
12\end{array}$ & $\begin{array}{c}\text { Pâturage } \\
19\end{array}$ \\
\hline $\begin{array}{l}\text { Lait }(\mathrm{kg} / \mathrm{j}) \\
\text { Bilan énergétique calculé (UFL/j) } \\
\text { Variation de note d'état corporel } \\
\text { Variation de lipides corporels (kg) }\end{array}$ & $\begin{array}{l}+2,2 \\
-0,6 \\
-0,2 \\
-4,2\end{array}$ & $\begin{array}{l}+\quad 2,7 \\
-\quad 1,6 \\
-\quad 0,4 \\
-12\end{array}$ & $\begin{array}{l}+\quad 2,0 \\
-\quad 0,5 \\
-13\end{array}$ \\
\hline
\end{tabular}

diminue fortement après la deuxième semaine et devient très faible en quatrième semaine, ce qui suggère que l'activité des formes de BST retard disponibles actuellement n'est pas constante durant la période qui sépare deux injections successives.

Les mêmes phénomènes cycliques existent, mais avec une moindre amplitude, dans le cas d'injections d'une demi-dose tous les 14 jours,c'est-à-dire pour une dose mensuelle totale comparable (Phipps 1987 et figure 1, Rémond et al 1988 - essai $\mathrm{n}^{\circ} 20$, Vérité et al 1989 et non publié - essai $n^{\circ} 3$, Bertrand et al 1989).

Les conséquences éventuelles des fluctuations de composition à l'échelle d'un troupeau sur la composition des laits collectés à l'échelle de micro-régions et/ou des unités de transformation de l'industrie laitière, devront être analysées par ailleurs en fonction du pourcentage de vaches traitées et de leur stade de lactation. Il en est de même pour la fréquence souhaitable des contrôles de qualité au niveau du tank de ferme en vue du paiement à la richesse en matières grasses et protéiques.

\section{5 / Discussion des résultats de production}

Il n'est pas possible d'estimer d'éventuelles variations de la réponse à la BST selon la race et le numéro de lactation, compte tenu du faible nombre d'essais disponibles, et réalisés dans des conditions non comparables. Dans 3 essais ( $\mathrm{n}^{\circ} 1,5$ et 11 ), la réponse des multipares tendait à être supérieure à celle des primipares, mais dans 3 autres $\left(n^{\prime \prime} 7,19,20\right)$, elle était inférieure ou égale.
- Il existe une grande variabilité des réponses (cumulées entre 2 injections) entre individus. Les valeurs individuelles sont difficiles à estimer pour des raisons méthodologiques liées à la difficulté de prendre en compte la persistance de la production laitière qu'aurait eu le même animal non traité. On peut donner une fourchette approximative de 0 à $+7 \mathrm{~kg} / \mathrm{j}$, pour une réponse moyenne de $3,5 \mathrm{~kg} /$ jour.

L'analyse de la variabilité individuelle a été effectuée dans les essais $n^{\circ} 1,2$ et 3 , où les injections ont eu lieu tous les 28 jours, ce qui permet d'évaluer la réponse maximum de chaque vache par rapport à la semaine précédant chaque injection (voir par exemple figure 2). Il apparaît alors que la réponse est répétable pour une même vache, intra- et inter-lactation (Vérité et al 1989 et non publié). La moitié environ de la variance intra-lactation de la réponse maximum est en effet expliquée par l'effet individu.

Il ne semble pas y avoir de lien entre la réponse d'une vache et son potentiel laitier antérieur au traitement. Ces différents résultats devront toutefois être confirmés sur de grands effectifs, en tenant compte des effets troupeaux, alimentation, stade de lactation, etc.

Les réponses plus faibles au pâturage qu'avec les rations hivernales peuvent s'expliquer soit par le stade de lactation (ou de gestation) plus avancé, soit par un effet saison ou environnement, soit par un effet nutritionnel, soit par une diminution du potentiel de réponse lorsque le traitement se prolonge, ou par plusieurs de ces facteurs à la fois.

Les effets du stade de lactation et de la saison paraissent peu probables car lorsque des traite- 
ment commencent à différents stades après le pic de lactation les réponses $(\mathrm{kg} / \mathrm{j})$ sont comparables (cf Chilliard 1988 a et b).

On peut donc penser à un effet de l'environnement (activité des animaux, etc) ou à une diminulion du potentiel de réponse (lié éventuellement au tissu mammaire, au stade de gestation, ou à la nécessité de reconstituer des réserves corporelles). Ceci concerne en particulier la dose de $24 \mathrm{mg} / \mathrm{j}$, avec laquelle les périodes pâturage faisaient suite à plusieurs mois de traitement BST, alors qu'avec la dose $35 \mathrm{mg} / \mathrm{j}, 6$ essais sur 8 ont commencé directement au pâturage.

Une interaction entre la BST et le niveau des apports nutritifs est souvent avancée pour expliquer les résultats inférieurs obtenus en France comparativement aux résultats obtenus aux USA, mais les données disponibles ne confirment pas toutes cette hypothèse. On ne dispose malheureusement pour estimer cette interaction que de comparaisons entre essais différents, ou entre périodes différentes d'un même essai, où toutes les conditions ne sont pas égales par ailleurs, ce qui limite très fortement la portée des hypothèses que l'on peut faire à partir de ces comparaisons.

Au pâturage, le supplément de lait dû à la BST est inférieur à celui obtenu avec des rations hivernales. Mais de nombreux facteurs interagissent : qualité de l'alimentation, durée du traitement, stade de lactation et de gestation, etc.
Ainsi les réponses obtenues aux USA avec des rations complètes en mélange à hautes teneurs énergétique et azotée sont plus élevées $(+5,3 \pm 2,0 \mathrm{~kg} / \mathrm{j}$, dans 10 essais, cf Chilliard $1988 \mathrm{~b}$ ), qu'avec les rations hivernales utilisées en France, (tableaux 4 et 5) avec distribution séparée du fourrage et du concentré, lequel est distribué en quantités limitées (cf tableau 3).

Dans 2 essais réalisés en France au pâturage (n) 5 et 19), on observe une diminution des réponses lors de perturbations climatiques ou de manque d'herbe en période estivale. Dans l'essai n" 19 (Lossouarn 1988), la réponse sur 17 vaches recevant de l'ensilage de maïs et pâturant une herbe de premier cycle, pendant 4 semaines, était de $4,2 \mathrm{~kg} / \mathrm{j}$. Cette réponse chute à $2,7 \mathrm{~kg}$ lors de la période suivante (25 vaches, 9 semaines) alors que les animaux ne recevaient plus d'ensilage et qu'une baisse de température freinait la pousse de l'herbe. Pendant la période suivante où l'herbe poussait correctement et où les vaches recevaient à nouveau de l'ensilage, la réponse n'a que peu augmenté et s'est stabilisée à $2,8-2,9 \mathrm{~kg}$. Lors de la rentrée à l'étable en fin de lactation (16 vaches, 6 semaines en moyenne avant le tarissement), la réponse est devenue très faible $(0,7 \mathrm{~kg} / \mathrm{j})$ malgré un niveau très élevé d'ingestion d'une ration de bonne qualité. Par ailleurs, l'absence de réponse dans l'essai $n^{\prime \prime} 4$ pourrait aussi être liée en partie à des conditions climatiques défavorables pour le pâturage.

Par contre, lors des 5 essais au pâturage réalisés par l'ITEB (n" 14 à 18), les vaches traitées recevaient la même quantité de concentré que les vaches témoins, bien que produisant plus de lail. Les réponses $(\mathrm{de}+1,6$ à $+4,1 \mathrm{~kg} / \mathrm{j}) \mathrm{ne}$ sont pas clairement liées aux différents facteurs nutritionnels connus différenciant les troupeaux. La réponse la plus faible a toutefois été obtenue dans un troupeau ne recevant pas d'ensilage en complément de l'herbe, et en état corporel initial médiocre.

On peut également remarquer que dans la majorité des essais au pâturage (10 cas sur 16) les animaux recevaient un complément de fourrages conservés (généralement de l'ensilage de maïs) qui aurait dû compenser en partie les éventuelles insuffisances du pâturage. Par ailleurs, dans l'essai n" 19 , le retour à une alimentation correcte n'a pas permis d'augmenter les réponses. D'autre part, la réponse moyenne de $+3,8 \mathrm{~kg} / \mathrm{j}$ obtenue dans 6 troupeaux d'Europe du Nord avec $35 \mathrm{mg}$ de BST/j et des rations hivernales riches en concentrés est du même ordre de grandeur que ce qui est observé dans les conditions françaises, avec généralement moins de concentré. Au sein des essais français on ne met pas en évidence de lien entre le rythme d'apport du concentré au lot témoin et la réponse à la BST.

Lebzien et al (1989) rapportent une réponse à $24 \mathrm{mg} / \mathrm{j}$ de BST retard égale à $+2,0 \mathrm{~kg} / \mathrm{j}$ dans un essai où le concentré était limité, et de $+3,6$ $\mathrm{kg}$ l'année suivante avec une distribution plus libérale. Ceci n'est toutefois pas confirmé par Vérité et al dans les essais $n^{\prime \prime} 1$ et 2 , où la réponse est plus faible $(2,0 \mathrm{vs} 2,7 \mathrm{~kg})$ avec le régime hivernal ayant la distribution la plus libérale d'aliment concentré.

En fait, le problème qui reste à résoudre est de montrer par des expériences comparant différents traitements nutritionnels quelle est l'importance réelle du facteur nutritionnel. Lors de quelques essais à court terme réalisés à l'étranger, il n'y a généralement pas eu de meilleure réponse à la BST en supplémentant l'animal en glucose, en acides aminés, en iso-acides ou en matières grasses (cf Chilliard 1988b). Dans les essais à long terme on connaît actuellement 3 comparaisons effectuées à l'étranger et 2 en France (tableau 7).

Dans trois essais où le niveau de concentré a été accru, on note une seule interaction positive avec la BST, dans le cas d'un régime à base d'ensilage de luzerne (Tessmann et al 1988). Lors de l'essai réalisé à Theix avec un ensilage de maïs d'ingestibilité moyenne $(17 \mathrm{~kg}$ de MS de ration totale ingérée pour $25 \mathrm{~kg}$ de lait/j), un apport supplémentaire de $2,9 \mathrm{~kg} / \mathrm{j}$ de concentré énergétique a été en grande partie compensé par une diminution de l'ingestion de fourrage, sans accroître la réponse à la BST (Rémond et al 1988, essai ${ }^{\circ} 20$ ). En outre dans un essai de Thomas et al (1987), la réponse était la même avec une ration complète en mélange, ou avec un régime hivernal avec distribution constante de concentré $(9 \mathrm{~kg} / \mathrm{j})$, bien que, dans ce dernier cas, le gain de poids des vaches traitées ait été inférieur de $20 \mathrm{~kg}$ en 24 semaines.

Chez les vaches recevant la BST, en augmentant fortement le taux de matières azotées de la ration et en abaissant leur dégradabilité dans le rumen, Mc Guffey et al (1988) observent un fort accroissement de la réponse chez des vaches hautes productrices, par rapport à un témoin " carencé » (les régimes enrichis en matières azotées peu dégradables ne concernant que les vaches ayant reçu de la BST, il est cependant 
Tableau 7. Réponses ( $\mathrm{kg}$ de lait/j) à la BST selon la nature du régime (essais à long terme).

\begin{tabular}{|c|c|c|}
\hline & Régime « bas » & Régime « haut » \\
\hline $\begin{array}{c}\text { Niveau de concentré } \\
40 \% \text { vs } 60 \% \text { concentré }(\mathrm{a})\end{array}$ & $+4,5$ & $+4,5$ \\
\hline 2,5 vs $5,4 \mathrm{~kg}$ concentré $/ \mathrm{j}(\mathrm{b})$ & $+2,7$ & $+2,7$ \\
\hline $12-32 \%$ vs $32-52 \%$ concentré $(\mathrm{c})$ & $+4,0$ & $+6,1$ \\
\hline $\begin{array}{c}\text { Niveau azoté } \\
14 \% \mathrm{P} \times \mathrm{HD} \text { vs } 17 \% \mathrm{P} \times \mathrm{BD}(\mathrm{d})\end{array}$ & $+2,4$ & $+5,3$ \\
\hline $15 \% \mathrm{P} \times \mathrm{HD}$ vs $15 \% \mathrm{P} \times \mathrm{BD}(\mathrm{e})$ & $+2,8$ & $+2,8$ \\
\hline
\end{tabular}

(a) Hemken et al 1988 (injections quotidiennes, $25 \mathrm{mg} / \mathrm{j}$ ).

(b) Rémond et al 1988 (BST retard, $35 \mathrm{mg} / \mathrm{j}$ ).

(c) Tessmann et al 1988 (injections quotidiennes, $25 \mathrm{mg} / \mathrm{j}$ ).

(d) Mc Guffey et al 1988 (BST retard, $24 \mathrm{mg} / \mathrm{j}$; P : Protéines, HD = dégradabilité élevée, BD = dégradabilité laible).

(e) Vérité et al 1989 (BST retard, $24 \mathrm{mg} / \mathrm{j}$ ).

difficile de savoir s'il y a interaction entre la BST et le régime). Ces auteurs notent en outre une faible persistance de la réponse à la BST avec le régime à faible valeur azotée. Par contre, Vérité et al (1989, essai $n^{\circ} 3$ ) obtiennent les mêmes réponses avec deux régimes à $15 \%$ de matières azotées mais de dégradabilités différentes (permettant une différence d'apport de $400 \mathrm{~g}$ de PDI par jour) et attribuent ce résultat au fait que les besoins en PDI étaient couverts avec les 2 régimes.

Il est donc actuellement difficile de se faire une idée des facteurs limitants de la réponse à la BST. Les comparaisons directes peuvent ne pas être concluantes car d'autres facteurs que ceux qui sont étudiés sont limitants : équilibre entre les différents nutriments, état corporel des animaux (une des plus faibles réponses en rations hivernales a été obtenue sur un troupeau à haut potentiel (essai $n^{\circ} 9$ ) mais en mauvais état corporel), « conditionnement» nutritionnel ou hormonal antérieur des réponses métaboliques... De nombreuses expériences seront sans doute nécessaires pour préciser ces différents aspects.

Enfin, il est possible que la mise en place d'une réponse rapide de la production laitière dans la semaine qui suit chaque injection, sans être accompagnée d'une augmentation correspondante de l'ingestion, se traduise par une diminution du bilan nutritionnel qui pourrait expliquer en partie la diminution des réponses au cours du temps, du fait des sollicitations répétées des mécanismes d'adaptation de l'organisme.

\section{6 / Effets sur la reproduction}

On peut distinguer les essais selon le stade de lactation auquel commencent les injections de BST : de 30 à 45 jours, de 60 à 70 jours, audelà de 85 jours.

a / Lorsque le traitement commence au-delà de 85 jours, une proportion importante des vaches est déjà fécondée et les effets de la BST sont plus difficiles à mettre en évidence, d'autant plus qu'à ce stade le niveau de production est déjà plus faible alors que le niveau d'ingestion est élevé, ce qui est favorable au succès de la reproduction. Dans 2 essais effectués en France et commençant en moyenne vers 85-90 jours de lactation, les performances de reproduction ne sont pas modifiées. On rapporte toutefois 2 cas d'avortement chez les vaches traitées.

\section{b / Dans les essais débutant vers la $9^{\circ}$ semaine} de lactation, les données disponibles sont plus nombreuses. Dans les études américaines, un retard de fécondation de 6-21 jours est rapporté (cf Chilliard 1988b). Ceci est confirmé par Bruneau et De Kerchove (1988) dans 4 troupeaux d'Europe de l'Ouest étudiés pendant 2 années consécutives (tableau 8). Les auteurs rapportent un retard à la fécondation de 13 jours la première année, sans modification du pourcentage de vaches non gravides. La deuxième année, ce pourcentage est plus élevé dans le lot BST, et l'on note un retard de 9 jours pour les fécondations réussies. L'effet de la BST sur l'intervalle vêlage - insémination fécondante (V - IF) semble être lié en partie à des mortalités embryonnaires précoces (essai 13). Le nombre d'inséminations par insémination fécondante (IA/IF) augmente quant à lui de $+0,3$ consécutivement au traitement par la BST. Ces effets seraient un peu plus importants que les chiffres cités cidessus si on les calculait sur les seules vaches non fécondées au momenl du début du traitement.

c / Avec des injections quotidiennes débutant dès la $5^{\text {e }}$ ou $6^{\text {e }}$ semaine de lactation, plusieurs auteurs signalent un allongement de l'intervalle vêlage - insémination fécondante (cf Chilliard 1988a). Cette tendance est confirmée en France avec la BST retard au cours de 3 ou 4 essais dont les résultats sont connus : + $16 \mathrm{j}$ sur l'intervalle $\mathrm{V}$ - IF, et + 0,3 IA/IF (tableau 9). Dans 2 de ces essais on rapporte 3 cas de veaux mortsnés ou morts dans les 48 heures sur 45 vêlages de vaches témoins, et 6 cas (dont $2 \times 1$ jumeaux) sur 42 vêlages de vaches traitées. 
Tableau 8. Effets de la BST administrée durant 2 lactations consécutives ( $36 \mathrm{mg} / \mathrm{j}$ ), à partir de la $9^{c}$ semaine. sur les performances de reproduction dans 4 troupeaux d'Europe de l'Ouest (Bruneau et De Kerchove 1.9881

\begin{tabular}{|c|c|c|c|c|}
\hline & \multicolumn{2}{|c|}{$1^{\text {re }}$ année } & \multicolumn{2}{|c|}{$2^{\mathrm{e}}$ année } \\
\hline & Témoin & BST & Témoin & BST \\
\hline Effectifs & 134 & 134 & 88 & 90 \\
\hline Vaches non gravides & $21(16 \%)$ & $20(15 \%)$ & $4(5 \%)$ & $13(14 \%)$ \\
\hline Intervalle $(V-I F)(a)$ & 96 & 109 & 95 & 104 \\
\hline $\mathrm{IA} / \mathrm{IF}(\mathrm{b})$ & 1,9 & 2,2 & 1,8 & 2,1 \\
\hline
\end{tabular}

(a) Intervalle vêlage - insémination fécondante, moyenne pondérée par le nombre de vaches gravides.

(b) Nombre d'inséminations artificielles / insémination fécondante.

Tableau 9. Effets de la BST (24 mg/j) administrée à partir de la $5^{\circ}$ ou $6^{\circ}$ semaine de lactation sur les performances de reproduction (essais français, résultats provisoires).

\begin{tabular}{|l|c|c|c|c|}
\hline \multicolumn{1}{|c|}{ Paramètre } & \multicolumn{2}{|c|}{ Intervalle V - IF } & \multicolumn{2}{c|}{ Nombre IA / IF } \\
\hline \multicolumn{1}{|c|}{ Lot } & Témoin & BST & Témoin & BST \\
\hline Nombre d'essais & 4 & 4 & 3 & 3 \\
Nombre de vaches gravicles & 65 & 59 & 50 & 44 \\
Moyenne pondérée & 95 & 111 & 1,5 & 1,8 \\
\hline
\end{tabular}

Si le traitement BST commence avant la fécondation, l'intervalle vêlage-insémination fécondante augmente de 9 à 16 jours, probablement en liaison avec la diminution du bilan énergétique des vaches en début de traitement.
Par ailleurs, dans un de ces 2 essais, on rapporte, les raisons étant inexpliquées, 4 avortements ayant eu lieu la même semaine chez les vaches traitées à la BST, contre un cas chez les témoins.

Les chiffres sur la mortalité et les avortements portent sur des effectifs trop faibles pour que des conclusions puissent être tirées.

\section{d / Les effets du traitement à la BST sur la} reproduction sont-ils équivalents à ceux de l'augmentation du niveau de production obtenu par sélection génétique? L'analyse des effets de ce facteur est complexe (cf Badinand 1983). Le récapitulatif d'un grand nombre d'études sur le sujet conduit aux hypothèses suivantes (Boichard 1987): lorsque le niveau de production des 3 premiers mois de lactation s'accroît de $1 \mathrm{~kg} / \mathrm{jour}$, le nombre d'IA/IF augmente au plus de 0,03 et l'intervalle $V-$ IF de 1,5 jour. Les effets de la BST sur la production lailière étant d'environ +2 à $+4 \mathrm{~kg} / \mathrm{j}$ en période hivernale (tableau 4), ceci correspondrait à des accroissements d'environ $0,1 \mathrm{IA} / \mathrm{IF}$, et de 3 à 6 jours de $\mathrm{V}$ - IF, qui sont inférieurs aux 0,3 IA et 9-15 jours de V - IF observés chez les vaches traitées à la BST avant 10 à 12 semaines de lactation (tableaux 8 et 9). La raison de cet " effet BST " réside probablement dans la forte diminution du bilan énergétique des animaux (figure 1) observée dans la quasi-totalité des études pendant les premières semaines du traitement.

Il convient de rester prudent dans l'interprétation des données de fécondité, dans la mesure où les effectifs sont assez restreints, où les données de base ne sont pas toujours accessibles et où les rapports sont très succincts dans ce domaine. Elles sont toutefois logiques et il est indiqué d'attendre la fécondation des vaches avant d'utiliser la BST, si l'on fixe comme objectif de maintenir l'intervalle vêlagevêlage du troupeau et cle ne pas accroître le nombre d'inséminations.

\section{7 / Santé et carrière des vaches}

L'état sanitaire des animaux traités par la BST ne semble pas être modifié lorsqu'on considère les différents lots d'animaux de chaque essai ou groupe d'essais. Il est toutefois évident qu'une étude statistique ne peut être faite que sur des effectifs importants, en regroupant les données individuelles d'un grand nombre d'essais. D'autre part, il conviendrait de le faire sur la base d'essais à long terme 3 ou 4 lactations successives avec des effectifs constants d'animaux) afin d'évaluer les effets cumulatifs et l'impact sur le taux de réforme des troupeaux. Il en est de même pour évaluer l'effet sur le potentiel des primipares. Il n'existe jusqu'ici que quelques essais publiés et réalisés à l'étranger sur 2 lactations successives, avec presque toujours une diminution importante $(30-40 \%)$ des effectifs entre la première et la deuxième année. De véritables essais à long terme seraient nécessaires, qui comptabiliseraient toutes les vaches entrées chaque année en expérience.

Un point important concernant la santé est celui du nombre de cellules du lait, puisqu'il intervient à la fois dans les plans de lutte contre les mammites et dans le paiement du lait à la qualité. Les cas de mammites cliniques ont été répertoriés dans 9 essais: 59 dans les lots témoins, et 76 dans les lots BST. L'analyse des cellules somatiques a été effectué dans 13 essais et on note 2 essais où ce paramètre est significativement plus élevé dans le lot BST, alors qu'il n'y a pas de différence dans 11 essais.

L'échantillon est beaucoup trop limité pour en conclure quoi que ce soit. Il semble d'ailleurs que les méthodes de calcul adéquates pour évaluer correctement l'effet d'un traitement sur le nombre de cellules du lait soient encore l'objet de discussions (cf rapport du Dr. Heeschen à la CEE) et il serait souhaitable de pouvoir regrouper les données individuelles 
des différents essais. On peut aussi remarquer que, de manière générale, l'élévation du niveau de production des vaches non traitées prédispose aux mammites (Barnouin et Karaman 1986).

\section{Conclusion}

Les données obtenues en France et à l'étranger suggèrent, en première approximation, que l'effet de la BST retard sur la production laitière varie assez nettement selon la dose totale injectée chaque mois, et assez faiblement selon la fréquence d'injection ( 1 ou 2 fois par mois).

Dans les 20 essais réalisés en France sur 360 vaches recevant de la BST retard tous les 14 ou 28 jours, à des doses correspondant à 24 ou 35 $\mathrm{mg} / \mathrm{j}$, les effets moyens ont été compris entre + 2,2 et $+3,7 \mathrm{~kg}$ de lait par jour avec des rations hivernales, et entre $+1,2$ et $+2,3 \mathrm{~kg}$ de lait par jour au pâturage. Les réponses inférieures au pâturage pourraient être dues à la qualité de l'herbe, au stade de gestation ou à la durée du traitement.

La composition du lait n'est pas modifiée au pâturage chez des vaches en milieu de lactation. Il existe une tendance à la diminution du taux protéique (- $0,5 \mathrm{~g} / \mathrm{l}$ environ) avec les rations hivernales distribuées avec un traitement à la BST pendant 3 à 4 mois après le pic de lactation. Cette conclusion tirée de 14 essais (tableau 4) amène à nuancer la tendance générale observée en moyenne sur toute la lactation au niveau international (cf ci-dessus). Il conviendrait done d'analyser statistiquement l'ensemble des données nouvellement disponibles depuis le 1/1/89, en dissociant les effets du temps écoulé depuis le début du traitement, du facteur alimentaire (énergie et protéines), et du stade de lactation (article en préparation).

Outre ces valeurs moyennes, la production et la composition du lait fluctuent entre deux injections successives, de façon croissante lorsque s'accroît l'intervalle entre injections.

Les vaches traitées montrent aussi une diminution de leur note d'état corporel $(-0,3$ point environ) lorsqu'elles sont traitées au pic de lactation (ce qui peut entraîner un retard de fécondation non négligeable), ou lorsqu'elles sont ultérieurement au pâturage avec des quantités limitées de concentré. Si l'on se fixe comme objectif de maintenir l'état corporel des vaches au vêlage suivant, le besoin nutritionnel des vaches traitées doit être couvert en fonction de l'accroissement de production laitière, comme chez les vaches non traitées.

Ce besoin nutritionnel peut éventuellement être couvert de façon différée (récupération du moindre dépôt de réserves corporelles après la fin du traitement).

La variabilité de la réponse est très grande entre essais et entre vaches. On connaît mal les facteurs qui la limitent. On peut supposer que la qualité et la quantité d'éléments nutritifs de la ration, ou l'état nutritionnel de l'animal avant traitement, jouent un rôle important, mais d'autres facteurs (génétiques, etc ) ne sont pas à exclure.
Les données actuellement publiées ne permettent pas d'évaluer d'éventuels effets à long terme sur la santé el la carrière des animaux.

Ce texte a été présenté à la journée d'information du 18 avril 1989 co-organisée par l'Instilut 'Technique de l'Elevage Bovin (ITEB) et le Syndicat de l'Industrie du Médicament Vétérinaire (SIMV).

\section{Références bibliographiques}

BADINAND F, 1983. Relations fertilité - niveau de pro dution-alimentation. Bull. Tech. CRZV Theix, INRA, 53, 73-83.

BARNOUIN J., el KARAMAN Z., 1986. Eıquêle éco pathologique continue : 9 . influence du nivedu de production sur la pathologie de la vache laitière. Ann. Rech. Vét., 17, 331-346.

BERTRAND et al, 1989. Etude d'une formulation de BST à effet prolongé pour la production laitière. Essais conduits dans 5 élevages sur des vaches lailières au pâturage. Compte rendu d'essais, ITEB, 149 rue de Bercy, 75012 Paris.

BOICHARD D., 1987. Analyse génélique des caractères de fertilité femelle chez les bovins lailiers. Thèse Docteur-Ingénieur, INA-P-G, 16 rue Claude Bernard, 75231 Paris Cedex 05.

BRUNEAU P., DE Kl:RCHOVE G., 1988. Somatotropine, reproduction et génétique chez la vache laitière. El. \& Ins., 228, 3-14.

CHILLIARI) Y., 1988a. Rôles et mécanismes d'action de la somalotropine (hormone de croissance) chez le ruminant en lactation. Reprod. Nutr. Dévelop., 28. 39-59.

CHILLIARD Y., 1988b. Review. L.ong-term effects of recombinant bovine somatotropin (rBST) on dairy cow performances. Ann. Zootech, 37 (3). 159-180.

CHILLIARD Y REMONI) B. CISSE M., COXAM V., 1989

Effects of slow released recombinant bovine somato tropin and concentrate allowance on dairy cow performances, body composition and blood metabolites and hormones. (7th. Int. Symp. Ruminant Physiol. Sendai. Japan), Asian-Australian J. Anim. Sci., 2, 497-498.

HEMKEN R.W., HARMON R.J., SIIVIA W.J., IIEHRSCHE G. EGGERT R.G., 1988. Response of lactating dairy cows to a second year of recombinant bovine somatrotropin (BST) when fed two energy concentrations. I. Dairy Sci. 71 (suppl. 1), 122 (Abstr.).

JOURNET M., CHILLIARD Y., 1985. Influence de l'al mentation sur la composition du lail. I. Taux butyreux facteurs généraux. Bull. Tech. C.R.Z.V. Theix, INRA, 60 $13-23$

KIRCHGFSSNER M., SCIIWAB W., MUFILLIR H.L. 1989. Fffect of bovine growth homone on energy metabolism of lactating cous in lono-term administration. Proc. 11th. Symp. Fnergy Metabol. (Lunteren. Netherlands, September 1988), EAAP Publ. n" 43, 143-146.

LEBZIEN P., ROHR K., DAENICKE R., SCIILUINSFN D. 1989. Recombinant somatotropin. A survey on a 2 years experimenl with dairy cows. In « CFC - Seminar on use of somatolropin in livestock production ". Bruxelles. 27 28 Septenber 1988. Flsevier Applied Science.

LOSSOUARN J., 1988. Etude d'une formulation retard de zinc-methionyl bovine somatotropine pour la production laitière. Compte rendu d'essai. I.N.A. Paris-Grignon Monsanto-France.

Mc BRIDE BW BURTON J.L BURTON I.H. 1988. Review. The influence of bovine growth hormone (somatotropin) on animals and their products. Res. Dev. Agric., $5,1-21$

Mc GUFFEY R.K. GREEN F.B. BASSON, R.P. 1988. Protein nutrition of the somatotropin-treated cow in early lactation. J. Dairy Sci.. 71 (suppl.1). 120 (Abstr.).

PEEL C.J., BAUMAN D.F., 1987. Somatotropin and lactation. J. Dairy Sci., 70, 474-486. 
PIIPPS R.II., 1987. The use of prolonged releatse bovine somatolropin in nulk production. [nt. Dairy led. Coms. (Ilelsinki, Finland) (2:3 p.).

RlinONil) B.. 1985. Influence de lablimentation sur la amposition du lail de vache - 2. Taux protégue : lac:leurs gencrimx. Bull. Fech. C.R.Z.V. Theix, INRA, 62. 5367.

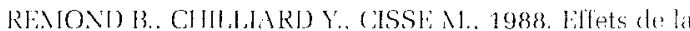
somatolmpine (sometribovis) sur l'ingestion, les perlormances ol le metibolisme des vaches labieres recevant beux niveanx d'aliments comentrés (INRA Tluix - Monsallo - Fancel.

TISSMANN N.J., KLEINMANS J., JIIMAN IR.R RAl)1.OFF H.J). SAl"I'JR L.D., 1988. Effecel of dielal'y foragr: grain palio on response of lactating daliry cons to recomGinant bovine somatotropin. J. Dairy Sici. Tl (suppl. 1). 121 (Absts.).

THOMAS C.. IOHINSSON H.D. FISIHLR W.J. BLOOM-

FIELD G.A. MORANT S.V. WILKINSON J.M.. 19887 bllect of somatoloppin on milk prochustion. resuroduction and healih of dairy cows. J. Dairy Sei., 70 (siuplul. 1), 175 (Abstr.).

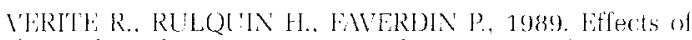
slow released somatolopin on dains cow pertormancas.

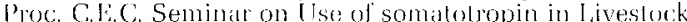
Production (Bruxelles, 27-29) september 19838). Flsovier Appliod Science. 